\title{
Stabilization of Plutonium in Subsursface Environments via Microbial Reduction and Biofilm Formation
}

\author{
Holden, Patricia \\ University of California, Santa Barbara \\ Neu, Mary P. \\ Los Alamos National Laboratory
}

\begin{abstract}
RESULTS TO DATE: Our work is towards mechanistically understanding interactions of unsaturated bacterial biofilms and their extracellular polymeric substances (EPS) with actinide metals and metal surrogates under vadose zone conditions. Because metal contaminants in the vadose zone co-occur with organic pollutants, some of our work has included experiments with organic pollutants.
\end{abstract}

In the past year, we studied interactions of Pseudomonas putida $\mathrm{mt}-2$, a gram negative soil organism frequently studied for bioremediation research, with $\mathrm{Cr}(\mathrm{VI})$. Working with pure cultures of $\mathrm{P}$. putida cultivated on membranes overlaying solid media, we observed that unsaturated biofilms rapidly and completely reduce $\mathrm{Cr}(\mathrm{VI})$ to $\mathrm{Cr}(\mathrm{III})$. $\mathrm{Cr}$ (III) accumulated outside the cells in EPS and on cells. EPS accumulation was enhanced when cells were exposed to $\mathrm{Cr}$. Cells were elongated when cultivated over hematite, whether dichromate was present or not. A key result of this study was that EPS glycosyl sugar composition changed upon exposure to $\mathrm{Cr}$, and glycosyl residues were more abundant upon $\mathrm{Cr}$ exposure. We hypothesized that enhanced proteins and glycosyl residues in the EPS were the result of cell lysis and retention of lysates outside the cell. This hypothesis was supported by comparing intracellular proportions of glycosyl residues and protein to extracellular proportions. However, extracellular DNA in the Cr-treated biofilms greatly exceeded the amount expected from cell lysis alone. We hypothesized that $\mathrm{Cr}$ (III) intercolation or sorption into DNA would be a mechanism for co-stabilization of $\mathrm{Cr}$ (III) and DNA outside the cells. Using dialysis tube studies, we showed that EPS treated with DNAse readily and completely liberated $\mathrm{Cr}$ (III) while untreated EPS did not. Coupled with our recent work that generically demonstrates the presence of extracellular DNA (eDNA) in unsaturated biofilms, our work suggests that a biotic fate for reduced $\mathrm{Cr}$ in the vadose zone is sorption to eDNA. This would be an important mechanism for stabilization of $\mathrm{Cr}(\mathrm{III})$ in vadose zone bacterial biofilms. The work was presented at the annual NABIR all investigator meeting and at the American Society for Microbiology general meeting in 2005, and has recently been submitted for publication in a peer-reviewed outlet.

This year we also completed a methods project related to this work and which improves the quality of our work on this project. The research concerns improving the visualization of bacterial biofilms using environmental scanning electron microscopy (ESEM). We researched and transferred staining methods used in conventional SEM and TEM for improving EPS resolution, and demonstrated that unsaturated (membrane and sand) and saturated aquatic biofilms were better and more equivalently resolved when a combination of stains and fixatives were employed. Importantly, ESEM preserves the delicate EPS matrix of unsaturated bioflms better than conventional SEM because specimen hydration is maintained. Also, variability in the performance of ESEM towards visualizing biofilms in the past might be attributable to the presence or absence of metals in the biofilm growth environment. Metal stains and conventional fixatives applied to biofilms prior to ESEM equalize the performance of the electron microscopy over all biofilms, independent of growth conditions. We were able to demonstrate this quantitatively through the use of a GIS-based image analysis approach that has previously only been applied to satellite imagery. The combination of ESEM, staining approaches, and this GIS-based image analysis method enabled us to quantify the roughening effect that biofilms have on otherwise smooth surfaces, and the smoothening effect biofilms have on otherwise rough surfaces. These methods and this understanding is broadly applicable in biofilm science. This work was conducted in collaboration with the Geography Department at UCSB. A manuscript for publication has recently been submitted for peer review. 
Other biofilm studies that will credit this project surround the effects of hydrophobic $\mathrm{C}$ sources and substrata hydrophobicity on the hydrophobicity of biofilm EPS and, in turn, bioavailability of hydrophobic $\mathrm{C}$ sources for biodegradation. This work is nearly completed and two manuscripts are in preparation for submitting for publication.

The personnel involved in the project include the PIs, and at UCSB a Research Associate, a Ph.D. student, and two undergraduate students. One of the two undergraduate students will begin an MS degree program with support of this project in Fall 2005. The other undergraduate will continue work on this project as an independent study student in Fall 2005.

DELIVERABLES: Two papers have recently been submitted for review and publication. The titles and abstracts are as follows:

1. John H. Priester, Allison M. Horst, Laurie C. Van De Werfhorst, Jose L. Saleta, Leal A. K. Mertes and Patricia A. Holden, "Enhanced Visualization of Microbial Biofilms by Staining and Environmental Scanning Electron Microscopy". Bacteria often grow as biofilms which are sessile structures consisting of cells and hydrated extracellular polymeric substances (EPS). Biofilms have important clinical and environmental implications, and their visualization at high resolution using electron microscopy can reveal much about their biology that is relevant to practice and research. Wet-mode environmental scanning electron microscopy (ESEM) of biofilms can offer advantages over conventional scanning electron microscopy (SEM) because, in ESEM, specimens can be imaged in their naturally-expanded state rather than dried which collapses the EPS matrix. EPS, however, is not electron dense and thus is not well-resolved with ESEM alone. Therefore, this study was undertaken to determine the applicability of several conventional SEM staining protocols for improving the resolution of biofilms and EPS using ESEM. Three different biofilm specimen types were used: 1) Pseudomonas aeruginosa unsaturated biofilms cultured on membranes, 2) P. aeruginosa cultured in moist sand, and 3) mixed communities developed on substrates suspended in an estuary. Working with the first specimen type, i.e. pure cultures of $P$. aeruginosa cultivated on membranes, an optimized staining regimen using ruthenium red, glutaraldehyde, osmium tetroxide and lysine was developed that enhanced biofilm topographic resolution for these otherwise very smooth samples. The efficacy of the approach was quantified using an image analysis tool that maps relief. When the optimized staining regime was used in ESEM imaging of sand cultures and aquatic biofilms, we quantified the smoothening effect that bacterial biofilms have on rough sand, and the roughening that aquatic biofilms impart on initially smooth coupons.

2. John H. Priester, Scott G. Olson, Samuel M. Webb, Mary P. Neu, Larry E. Hersman, Patricia A. Holden. "Enhanced Exopolymer Production and Chromium Stabilization in Pseudomonas putida Unsaturated Biofilms" Chromium-contaminated soils threaten surface and groundwater quality at many industrial sites. In vadose zones, indigenous bacteria can reduce $\mathrm{Cr}(\mathrm{VI})$ to $\mathrm{Cr}(\mathrm{III})$ but the subsequent fate of $\mathrm{Cr}$ (III) and the roles of bacterial biofilms are relatively unknown. To investigate, we cultured Pseudomonas putida, a model organism for vadose zone bioremediation, as unsaturated biofilms on membranes overlaying iron-deficient solid media either containing molecular dichromate from potassium dichromate (Cr-only treatment) or with deposits of solid, dichromate-coated hematite (Fe+Cr treatment) to simulate vadose zone conditions. Controls included iron-deficient solid media and an Fe-only treatment using solid hematite deposits. Under iron-deficient conditions, chromium exposure resulted in lower cell yield and lower amounts of cellular protein and carbohydrate, but providing iron in the form of hematite overcame these toxic effects of $\mathrm{Cr}$. For the $\mathrm{Cr}$ and $\mathrm{Fe}+\mathrm{Cr}$ treatments, $\mathrm{Cr}(\mathrm{VI})$ was completely reduced to $\mathrm{Cr}$ (III) that accumulated on biofilm cells and EPS. Chromium exposure resulted in elevated extracellular carbohydrates, protein, DNA, and EPS sugars that were relatively enriched in $\mathrm{N}$-acetyl-glucosamine, rhamnose, glucose and mannose. The proportions of EPS protein and carbohydrate relative to intracellular pools suggested $\mathrm{Cr}$ toxicity-mediated cell lysis as the origin. However, DNA accumulated extracellularly in amounts far greater than expected from cell lysis, and $\mathrm{Cr}$ was liberated when extracted EPS was treated with DNase. These results demonstrate that $\mathrm{Cr}$ accumulation in unsaturated biofilms occurs with enzymatic reduction of $\mathrm{Cr}(\mathrm{VI})$, cellular lysis, cellular association, and extracellular DNA binding of $\mathrm{Cr}(\mathrm{III})$ which altogether can facilitate localized biotic stabilization of $\mathrm{Cr}$ in contaminated vadose zones. 
COLLABORATIONS: This application is a collaboration with Los Alamos National Laboratory (Lead PI, M.P. Neu, LANL). 